Ann. Zootech., I977, 26 (r), I3I-I38.

Note

\title{
Note sur les variations \\ du taux d'acides gras non estérifiés plasmatiques chez la Vache à la fin de la gestation
}

\author{
M. PETIT * ct. B. REMOND ** \\ avec la collaboration technique de G. Bourlot *, \\ G. Triliat * et Maryse Vérité ** \\ * Labovatoire de la Production de Viande, \\ ** Laboratoive de la Production Laitiève, \\ Centre de Recherches de Clermont-Ferrand, I.N.R.A. \\ Theix - St-Genès-Champanelle \\ 63110 Beaumont (France)
}

\begin{abstract}
Résumé
Trente-sept vaches de race Charolaise, Aubrac ou Salers ont été réparties en 2 lots et ont reçu 2 niveaux d'alimentation énergétique différents.

Pendant les I I dernières semaines de gestation ( 182 mesures au total) nous avons mesuré l'évolution de la teneur du plasma en acides gras non estérifiés.

La teneur du plasma en acides gras non estérifiés a été plus élevée chez les vaches qui avaient reçu le bas niveau d'alimentation que chez celles qui avaient reçu le haut niveau, et chez les Charolaises que chez les Aubrac et les Salers. Elle a augmenté linéairement avec le stade de gestation $\epsilon$ t le poids des veaux à la naissance (tabl. I et 2, et fig. I).

- La variabilité résiduelle élevée des résultats et l'effet, semble-t-il, d'autre(s) facteur(s) que le bilan énergétique limitent l'intérêt de la mesure de la teneur du plasma en acides gras non estérifiés pour l'appréciation de l'état nutiitionnel des vaches pendant la fin de la gestation.
\end{abstract}

\section{Introduction}

La teneur du plasma en acides gras non estérifiés permet d'apprécier en partie l'état de nutrition énergétique des Ruminants (cf. revue de BowDEN, r97I). Cette mesure est particulièrement intéressante car les variations du poids vif, qui sont souvent utilisées pour mesurer l'état nutritionnel, ne tiennent pas compte des 
modifications de la composition corporelle, et chez le Ruminant, de la variabilité importante du poids des contenus digestifs. Son intérêt est encore accru à la fin de la gestation, en raison de l'augmentation importante du poids des produits de la conception qui masque les modifications du poids vif vide de la mère.

La teneur du plasma en acides gras non estérifiés a d'ailleurs été utilisée pour apprécier l'importance et l'évolution du bilan énergétique à la fin de la gestation, en particulier chez la brebis (ReId et HIN KS, I962; Russel, Doney et ReID, I967; Russel et Doney, I969) mais aussi plus récemment chez la vache (BowdEN, I974).

Lors d'une étude sur les possibilités de réduire le niveau d'alimentation énergétique de vaches de races rustiques ou à viande au cours de la gestation, nous avons observé l'évolution de la teneur du plasma sanguin en acides gras non estérifiés durant les 2 à 3 derniers mois prépartum.

\section{Matériel et méthodes}

On a utilisé $\mathrm{x} 4$ vaches Charolaises, I 2 vaches Aubrac et II vaches Salers, toutes multipares et âgées de 4 ans ou plus, qui étaient apparemment en très bon état corporel au début de la période expérimentale ( $\mathrm{I} \pm 2$ semaines avant le vêlage). Les vaches de chaque race ont été réparties en 2 lots sur la base de leurs poids vif, date de vêlage et état apparent.

L'un des lots (lot témoin : T) a reçu chaque jour la quantité d'énergie correspondant approximativement aux besoins moyens d'entretien et de gestation (d'après MOE et TYRELL, I972) au cours de cette période : I5I \pm 7 kcal d'énergie métabolisable par $\mathrm{kg}$ de poids métabolique. L'autre lot (lot bas : B) a reçu 83 p. roo de la quantité d'énergie apportée au lot T, soit $126 \pm 4$ kcal. La quantité d'énergie offerte à chaque vache a été calculée à partir de son poids vif obtenu lors des pesées effectuées 3 jours consécutifs juste avant la période expérimentale $(623 \mathrm{~kg}$ en moyenne pour chacun des 2 lots). Les rations, exclusivement composées de fourrages longs (foin ou foin + paille) et d'un mélange de minéraux, ont été distribuées individuellement et en quantité constante jusqu'au vêlage, 2 fois par jour, à 8 et I7 heures. Les teneurs en énergie métabolisable des fourrages ont été estimées à partir de la digestibilité de l'énergie de ces fourrages obtenue avec des moutons adultes en cage à bilan et nourris ad libitum; on a supposé que le rapport énergie métabolisable

énergie digestible était égal à 82 p. ıoo.

Toutes les 2 semaines, juste avant la distribution des aliments du matin, on a prélevé du sang dans la veine jugulaire. Le nombre de prélèvements a varié de 4 à 7 selon les vaches, et tous les prélèvements ont été effectués entre 98 et 2 jours avant la mise bas. Le sang était centrifugé immédiatement et le plasma stocké au congélateur $\left(-20^{\circ} \mathrm{C}\right)$ jusqu'à l'analyse. La teneur en acides gras non estérifiés (A.G.N.E.) a été mesurée par la méthode de Dole et Meinertz (I960).

Les variations du taux d'A.G.N.E. ont été exploitées statistiquement par une analyse de variance et covariance non orthogonale (SEEBECK, I973), tenant compte à la fois de la race, du niveau d'alimentation et du stade de gestation. Seules ont été prises en compte dans cette analyse les vaches gestantes d'un seul veau. Nous 
avons ensuite étudié l'influence du poids à la naissance des veaux sur la teneur moyenne en A.G.N.E. des plasmas obtenus lors des deux derniers prélèvements précédant le vêlage (effectués entre 2 et 23 jours avant la parturition).

\section{Résultats et discussion}

\section{Infuence du niveau d'alimentation}

La différence d'apport énergétique entre les 2 lots n'a pas eu d'influence significative sur le poids des veaux à la naissance qui a été de $4 \mathrm{I}, 6 \mathrm{~kg}$ dans le lot des vaches les mieux alimentées (lot $\mathrm{T}$ ) et de 40,0 kg dans le lot de celles qui l'étaient moins bien (lot $B$ ), ni sur l'évolution du poids vif des mères qui ont perdu respectivement $\mathrm{r} 9,3$ et $2 \mathrm{I}, 3 \mathrm{~kg}$ entre la pesée précédant l'expérience et celle suivant le vêlage. Ces variations de poids sont difficilement interprétables, en raison du poids des produits de la conception au début de la période expérimentale, des modifications possibles de la composition corporelle, et surtout des variations importantes

\section{TABI}

Influence du nivcau d'alimentation, du stade de gestation et de la race sur le taux d'acides gras non estérifiés plasmatiques de vaches gestantes

Effects of the level of feeding, stage of pregnancy and breed on the concentration of plasmatic non esterified fatty acids

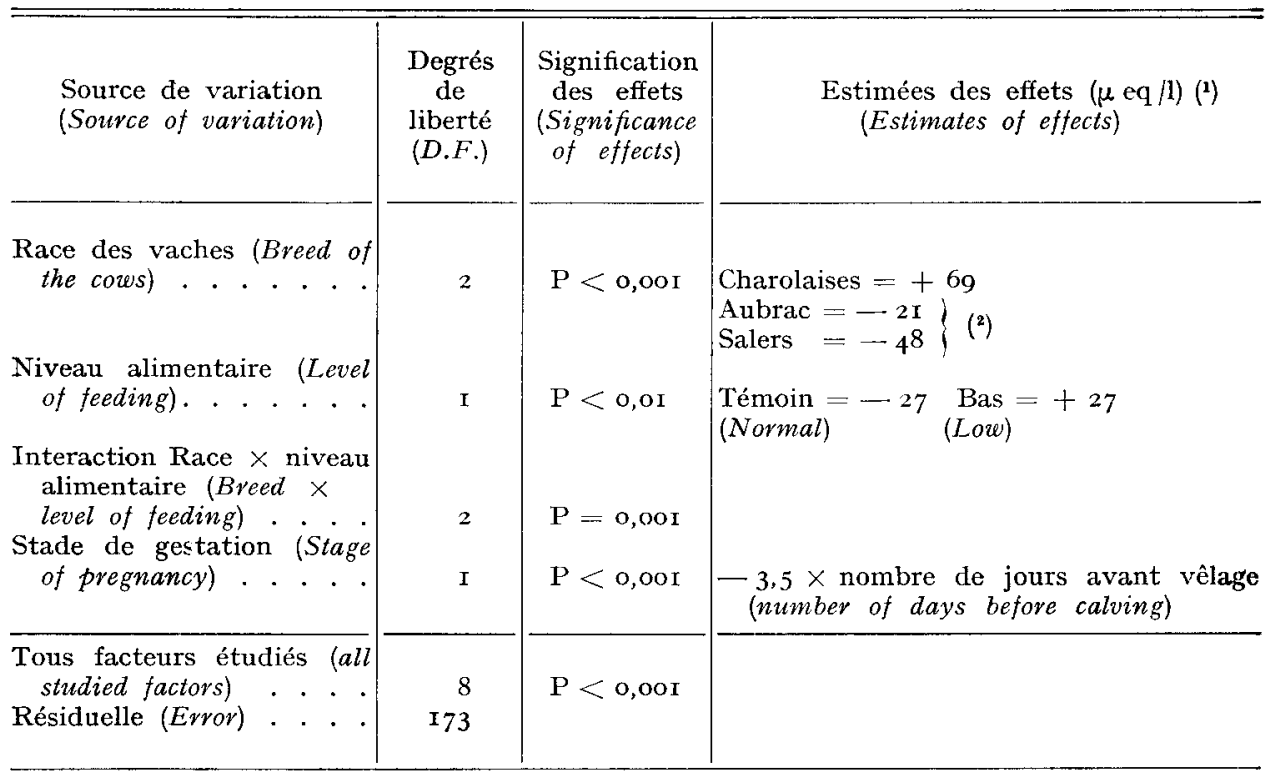

1) Ira valeur moyenne, corrigée pour l'ensemble des facteurs étudiés, est égale à $366 \mu$ eq $/ 1$ (Mean value corrected for all the studied factors, is $366 \mu$ eq $(l)$.

(2) La différence entre les Aubrac et les Salers r'est pas significative. (The difference between Aubrac and Salers cows is non significant.) 
du contenu digestif, en particulier autour du vêlage. Cependant, le taux d'A.G.N.E. des vaches du lot B a été significativement supérieur (de $54 \mu \mathrm{eq}$. par litre, soit I6 p. roo) à celui des vaches du lot $\mathrm{T}$ (tabl. I).

\section{Influence du stade de gestation}

Le taux d'A.G.N.E. a augmenté tout au long de la période expérimentale, de façon parallèle et statistiquement linéaire dans les 2 lots : de $3,5 \mu \mathrm{eq} / 1$ par jour de gestation (tabl. I) (fig. I). Mais cet accroissement n'a pas reflété celui des besoins énergétiques (et donc du bilan), puisque, selon MoE et TyRELL (I972), ceux-ci augmentent de façon exponentielle au cours des 3 derniers mois qui précèdent le vêlage. Comme l'ont déjà suggéré Sykes et FIELD (I972), il est possible que les changements hormonaux qui ont lieu à la fin de la gestation influencent la teneur du plasma en A.G.N.E. Nos résultats sont semblables à ceux de Bowden (I974), qui a cependant observé sur des vaches Aberdeen-Angus multipares des teneurs en A.G.N.E. sensiblement plus élevées malgré des apports énergétiques plus importants.

\section{Infuence de la race}

Le taux d'A.G.N.E. a été significativement plus élevé chez les Charolaises que chez les Aubracs et Salers, respectivement de 9o et $\operatorname{\text {II}} \mu \mu$ eq par litre (tabl. I). Cela ne semble pas dû̀ à un stress plus important lors des prises de sang, ni à une sous-alimentation plus prononcée chez les Charolaises : l'évolution du poids vif au cours de la période expérimentale a été en moyenne la même pour les trois races et le poids des veaux à la naissance, rapporté au poids métabolique de leurs mères a été égal à $0,33,0,35$ et 0,32 pour les Charolaises, Aubracs et Salers respectivement.

Il est donc possible que le niveau "normal d'A.G.N.E. plasmatiques soit plus élevé chez les Charolaises; les teneurs mesurées chez les vaches du lot $T$ avant le $5^{0^{e}}$ jour précédant le vêlage (c'est-à-dire pendant la période où les apports théoriques d'énergie étaient supérieurs aux besoins), ont été de 305 et $259 \mu$ eq $/ 1$ respectivement pour les Charolaises et les Salers + Aubrac. Russel et Doney (Ig69) et HALLidAy et al. (I969) ont déjà observé chez la brebis, d'importantes variations raciales dans le taux d'A.G.N.E. plasmatiques; en revanche, Williams et al. (I963) n'ont pas observé de différences entre des vaches Jersey et Holstein, ni BowdeN (I974) entre des vaches Aberdeen-Angus et Hereford.

La différence entre les teneurs en A.G.N.E. des lots B et T, qui a été importante chez les vaches Charolaises ( $145 \mu \mathrm{eq} / 1$ ) et faible chez les vaches Aubrac et Salers (respectivement - I4 et $29 \mu \mathrm{eq} / \mathrm{l}$ ), peut être due au fait que chez les vaches Charolaises l'effet de la sous-alimentation appliquée au lot $\mathrm{B}$ a été accentuée par un poids de veau moyen à la naissance plus élevé que dans l'autre lot (respectivement 43,2 et $39,5 \mathrm{~kg}$ ), alors que chez les vaches Aubrac et Salers, l'effet de la sousalimentation a été au moins en partie compensé par un poids de veau à la naissance plus faible (lot B : $37,7 \mathrm{~kg}$ et lot $\mathrm{T}: 42,4 \mathrm{~kg}$ ).

La moyenne des A.G.N.E. corrigée pour l'ensemble des facteurs étudiés dont 


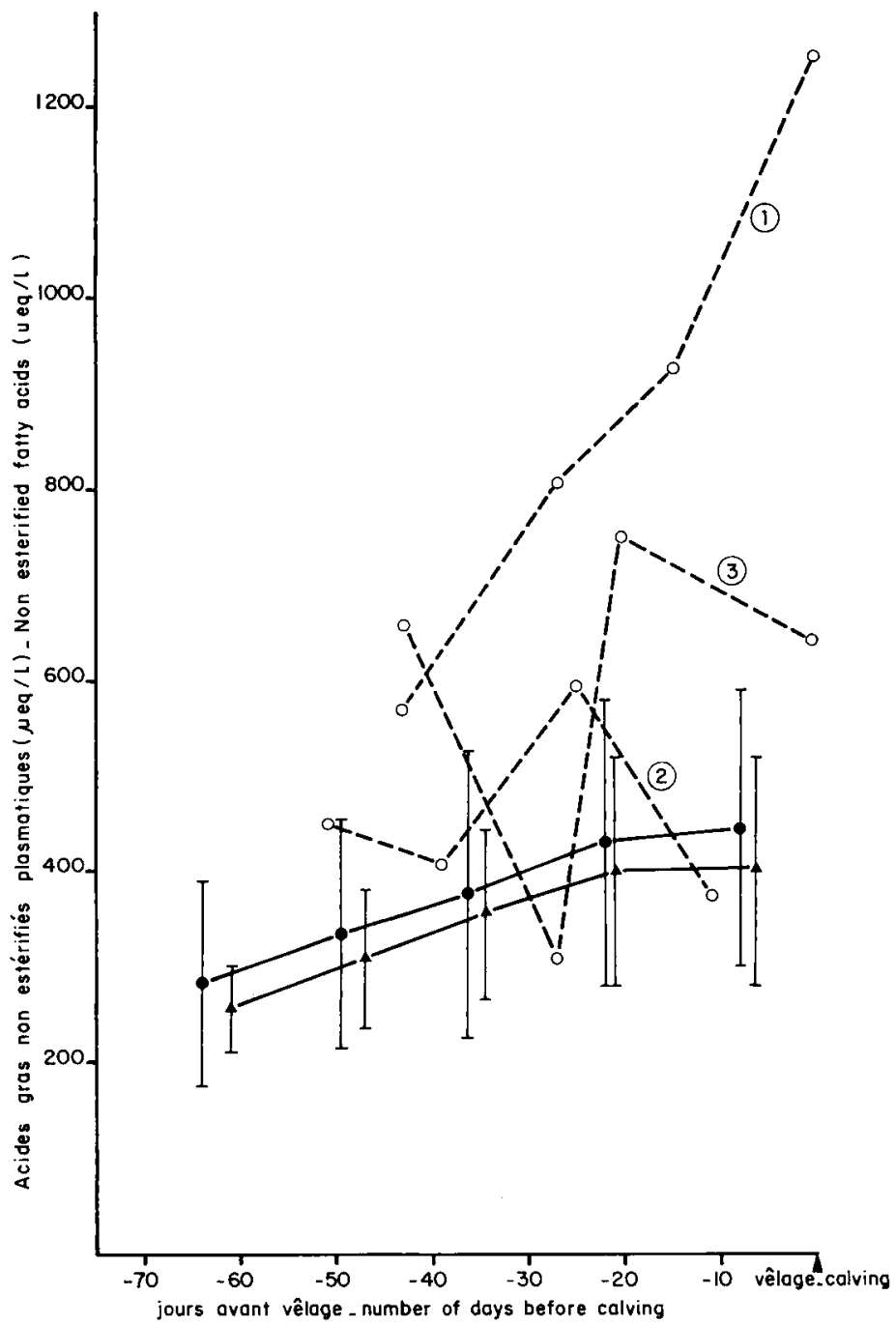

FIG. I. - Evolution du taux plasmatique des acides gras non estérifiés des vaches en gestation Evolution of the concentration of plasmatic non esterified fatty acids during late pregnancy

Nota : Les traits verticaux indiquent l'écart-type total de part et d'autre de la moyenne. (Vertical lines show standard deviation both sides of the mean.)

Trois vaches gestantes de deux veaux ont reçu un bas niveau d'alimentation : deux Charolaises ( $\mathrm{I}$ et 2) et une Salers (3), dont les poids des portées étaient respectivement $49,6,7 \mathrm{I}, 8$ et $56,9 \mathrm{~kg}$. (Three cows bearing twins were given a low level of feeding $=$ two Charolais cows (I and 2). and one Salers cow (3). Total birth weights of the litters were respectively 49.6, 71.8 and $56.9 \mathrm{~kg}$ ).

la race des vaches, le niveau alimentaire et le stade de gestation est de $366 \mu \mathrm{eq} / \mathrm{l}$, et l'écart-type résiduel correspondant est de I23 $\mu$ eq/l. Les facteurs retenus permettent d'expliquer $44 \mathrm{p}$. roo de la variance totale. 


\section{Influence du poids des veaux à la naissance}

Il existe une liaison hautement significative entre le taux d'A.G.N.E. moyen obtenu lors des deux derniers prélèvements sanguins précédant la mise bas et le poids des veaux à la naissance (tabl. 2). Lorsque le poids des veaux à la naissance

\section{TABLEAU 2}

Influence du poids des veaux à la naissance sur le taux moyen d'acides gras non estérifiés obtenu au cours des 2 à 3 dernières semaines de gestation

Effect of the birth weight of the calves on the average concentration of non esterified plasmatic fatty acids during the last 2 or 3 weeks of pregnancy

\begin{tabular}{|c|c|c|c|}
\hline $\begin{array}{l}\text { Source de variation } \\
\text { (Source of vaviation) }\end{array}$ & $\begin{array}{l}\text { Degrés } \\
\text { de } \\
\text { liberté } \\
(D . F .)\end{array}$ & $\begin{array}{l}\text { Signification } \\
\text { des effets } \\
\text { (Significance } \\
\text { of effects) }\end{array}$ & $\begin{array}{c}\text { Estimées des effets }(\mu \text { eq } / \mathbf{l}) \\
(\mathbf{1}) \\
(\text { Estimates of effects })\end{array}$ \\
\hline 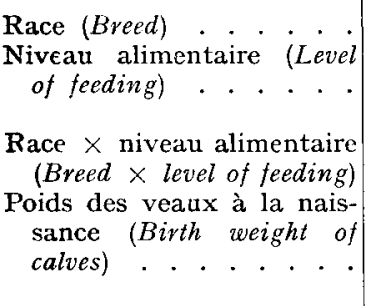 & I & $\begin{array}{c}\text { NS }\left({ }^{2}\right) \\
(P<0, \mathrm{I}) \mathrm{NS} \\
\text { NS }\end{array}$ & 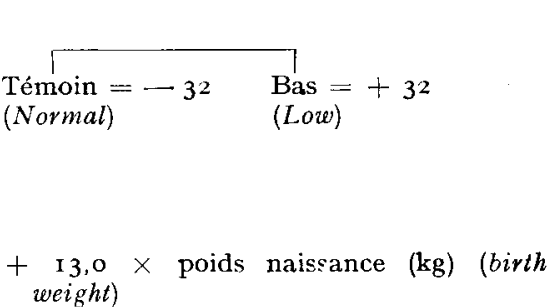 \\
\hline 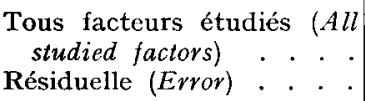 & $\begin{array}{l}10 \\
23\end{array}$ & $\mathrm{P}<0,0 \mathrm{I}$ & \\
\hline
\end{tabular}

(') Ira valeur moyenne, corrigée pour l'ensemble des facteurs étudiés, est égale à 4 I $5 \mu$ eq $/ 1$ (The mean value, corrected for all studied factors, is $4 I 5 \mu \mathrm{eq} / \mathrm{l}$.)

(2) NS = Non Significatif.

passe de 35 à $45 \mathrm{~kg}$, l'estimée du taux d'A.G.N.E. lors des deux à trois dernières semaines de la gestation passe de 350 à $480 \mu$ eq/l. Le déficit énergétique est donc sensiblement accru pour les vaches portant des veaux lourds. D'ailleurs, leur perte de poids entre la triple pesée précédant le début de l'expérience et la pesée 4 jours après le vêlage est linéairement liée au poids des veaux à la naissance $(r, 37 \times$ poids naissance).

Même en tenant compte dans cette analyse statistique du poids à la naissance des veaux, l'écart-type résiduel du taux d'A.G.N.E. reste important $(96 \mu \mathrm{eq} / \mathrm{l})$, bien que les valeurs utilisées correspondent à la moyenne des deux dernières mesures. Il en résulte que la race des vaches et le niveau d'alimentation n'ont plus d'effet significatif dans cette analyse (tabl. 2) où l'ensemble des facteurs de variation retenus explique 53 p. Ioo de la variance totale des A.G.N.E. 
On peut en outre, remarquer que trois vaches supplémentaires qui ont mis bas des jumeaux dans le lot B, ont eu des taux d'A.G.N.E. en moyenne plus élevés que chez les vaches gestantes d'un seul veau.

\title{
Conclusion
}

Comme chez la brebis gestante, une augmentation du taux d'A.G.N.E. traduit un accroissement du déficit énergétique, qu'il soit dû à un plus faible niveau moyen d'alimentation, à un stade de gestation plus avancé, à un poids de fotus supérieur. Cependant, l'évolution du taux d'A.G.N.E. au cours des toutes dernières semaines de gestation ne reflète pas, dans cette étude, l'augmentation exponentielle des besoins. Par ailleurs, il semble exister des différences raciales importantes qu'il reste à préciser et à expliquer. Enfin, pour un même apport d'énergie par kg de poids métabolique et un même poids de veau à la naissance, il reste une grande variabilité résiduelle qui peut être due pour partie à la méthode de mesure, mais aussi à des différences individuelles. Cela limite l'intérêt de cette mesure pour estimer l'état nutritionnel des vaches à la fin de la gestation.

Rę̧u pour publication en octobre 1976.

\begin{abstract}
Summary
Variations in the plasma level of non-esterified fatty acids in the cow during late pregnancy
\end{abstract}

Thirty seven cows of the Charolais, Aubrac or Salers breed were distributed into 2 groups and received diets of 2 different energy levels.

The evolution in the plasma level of non-esterified fatty acids was measured during the last II weeks of pregnancy (a total of 182 measurements).

The plasma content of non-esterified fatty acids was higher in cows receiving the low energy diet than in those fed with the high one, and more elevated in Charolais than in Aubrac and Salers. It increased linearly with the stage of pregnancy and the weight of calves at birth (Tables $I$ and 2 , and figure).

The high residual variability of the results and the possible effect of another or other factor(s) than the energy balance reduce the interest of using the measure of the plasma level of nonesterified fatty acids for estimating the nutritional status of cows during late pregnancy.

\section{Références bibliographiques}

Bowden D. M., I97I. Non-esterified fatty acids and ketone bodics in blood as indicators of nutritional status in ruminants: a review. Can J. anim. Sci, , 51, I-I3.

Bowden D. M., I974. Blood metabolites as indicators of energy status of cows. 6th Symposium on Energy metabolism of farm animals. K. H. Menke, H. J. Lantzsch, et J. R. Reichl, Éditeurs. Stuttgart, p. 59-62.

Dole V. P., Meinertz H., I96o. Determination of long-chain fatty acids in plasma and tissues. J. Biol. Chem., 235, 2595-2599.

Halliday R., Sykes A. R., Slee J., Field A. C., Russel A. J. F., rg69. Cold exposure of Southdown and Welch Mountain sheep. 4-Changes in concentration of free fatty acids, glucose, acetone, protein-bound iodine, protein and antibody in the blood. Anim. Prod., 11, 479-491. 
Moe P. W., Tyrrell H. F., r972. Metabolizable energy requirements of pregnant dairy cows. J. Dairy Sci., 55, $480-483$.

Reid R. L., Hinks N. T., I962. Studies on the carbohydrate metabolism of sheep. Aust. J. Agric. Res., 13, I I I 2-I I 23.

Russel A. J. F., Doney J. M., Reid R. L., I967. The use of biochemical parameters in controling nutritional state in pregnant ewes, and the effect of under-nourishment during pregnancy on lamb birth-weight. J. Agric. Sci., 68, 35I-358.

Russel A. J. F., DoNey J. M., I969. Obscrvations on the use of plasma free fatty acid concentrations in the determination of maintenance requirements of sheep. J. Agric. Sci., 72, 59-63.

SeEBEck R. M., I973. The effect of body weight loss on the composition of Braham and Africander cross steərs. I-Empty body weight, dressed carcass weight, and offal components. J. Agric. Sci., 80, 20I-2 I0.

Sykes A. R., Freld A. C., 1972. Effects of dietary deficiencies of energy, protein and calcium on the pregnant ewe. III- Some observations on the use of biochemical parameters in controlling energy undernutrition during pregnancy and on the efficiency of utilization of energy and protein for foetal growth. J. Agric. Sci., 78, I27-133.

Williams W. F., Lee S. D., Head H. D., LyNCH J., i963. Growth hormone effects on bovine blood plasma fatty acid concentration and metabolism. J. Dairy Sci., 46, I405-I408. 